\title{
Implicações do bullying na saúde mental de adolescentes obesos: revisão integrativa
}

\author{
Delbana Pereira Rodrigues \\ Marianne Lira de Oliveira \\ Paula de Moura Lopes \\ Cássio Eduardo Soares Miranda
}

\section{RESUMO}

A violência é um problema de saúde pública crescente, que traz sérias consequências individuais e sociais. Nesse contexto, destaca-se a prática de bullying relacionado à obesidade. Não é incomum indivíduos considerados obesos sofrerem maior exclusão social e apresentarem quadros de ansiedade, privação e problemas de comportamento alimentar que se agravam com a prática do bullying. $\mathrm{O}$ objetivo deste artigo é aprofundar a discussão a respeito das implicações do bullying na saúde mental de adolescentes obesos. Trata-se de um estudo de revisão integrativa realizada nas bases eletrônicas: Biblioteca Virtual em Saúde (BVS), United States National Library of Medicine (PubMed); e Scientifc Electronic Library Online (SciELO). Do total de estudos incluídos na revisão final $(\mathrm{n}=05), 02$ foram realizados no Brasil, 01 no Reino Unido, 01 nos Estados Unidos e 01 na Noruega. Foi possível observar a existência de sentimentos negativos vivenciados por adolescentes obesos, vítimas de bullying, tais como ansiedade, tristeza, angústia e sentimento de exclusão social. Tais achados evidenciam uma certa urgência para o desenvolvimento de ações permanentes de enfrentamento ao bullying e promoção do convívio saudável.

Palavras-chave: Adolescentes; Bullying; Obesidade; Saúde mental.

\section{ABSTRACT \\ Implications of bullying in the mental health of obese adolescents: integrative review}

Violence is a growing public health problem that brings serious individual and social consequences. In this context, it stands out the practice of bullying related to obesity. It is not uncommon that obese individuals suffer more from social exclusion and present anxiety issues, eating deprivation and behavior problems that aggravate the bullying. The objective of this article is to deepen the discussion about the implications of bullying on the mental health of obese adolescents. It is a study of integrative review performed in electronic databases: Virtual Health Library (VHL), United States National Library of Medicine (PubMed); and Scientifc Electronic Library Online (SciELO). The total number of studies included in the review end $(n=05), 02$ were conducted in Brazil, 01 in the United Kingdom, 01 in the United States and 01 in Norway. It was possible to observe the existence of negative feelings experienced by obese adolescents, victims of bullying, such as anxiety, sadness, anguish and feelings of social exclusion. Such findings show a certain urgency for the development of permanent actions to fight against bullying and promote a healthy living.

Keywords: Adolescents; Bullying; Obesity; Mental health.

A violência é um problema de saúde pública crescente, que traz sérias consequências individuais e sociais. Nesse sentido, o bullying (termo oriundo da palavra inglesa bully - valentão, brigão) é defınido como uma forma de violência repetitiva, com ou sem a intenção de causar dor ou desconforto, manifestado através de gestos, palavras, atitudes e/ou comportamentos que

\section{Sobre os autores}

D. P. R.

http://orcid.org/0000-00023349-2537

Universidade Federal do PiauíTeresina, PI

delbanarodrigues@ifma.edu.br

M. L. O.

http://orcid.org/0000-0001-

5335-1806

Universidade Federal do PiauíTeresina, PI

marianne-lira.15@hotmail.com

P. M. L.

http://orcid.org/0000-0002-

2106-3743

Universidade Federal do PiauíTeresina, PI

p.demouralopes@gmail.com

\section{E. S. M.}

http://orcid.org/0000-0002-

8990-1205

Universidade Federal do PiauíTeresina, PI

cassioufpi@gmail.com

\section{Direitos Autorais}

Este é um artigo de acesso aberto e pode ser reproduzido livremente, distribuído, transmitido ou modificado, por qualquer pessoa desde que usado sem fins comerciais. 0 trabalho é disponibilizado sob a licença Creative Commons CC-BY-NC.

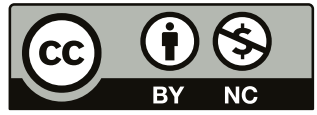




\section{n* INTERACÃo EM PSICOLOGIA}

atentem contra a dignidade e a integridade física e/ou psíquica de um indivíduo, causando-lhe medo, insegurança, dor, angústia e sofrimento (Bolzan Berlese, et al., 2017; Costa, et al., 2012).

Por ser um problema descrito em vários países, a OMS tem coordenado pesquisas de monitoramento do bullying, com o objetivo de identificar mudanças e apoiar políticas para o seu enfrentamento. De acordo com o relatório da Organização das Nações Unidas para a Educação, a Ciência e a Cultura (UNESCO), estima-se que, anualmente, 246 milhões de crianças e adolescentes no mundo são vítimas de violência escolar e bullying. No Brasil, a Pesquisa Nacional de Saúde do Escolar (PeNSE) mostrou um aumento expressivo na ocorrência desses eventos entre adolescentes. As prevalências apresentadas foram de: 5,4\% em 2009; 6,8\% em 2012 e 7,4\% em 2015 (Malta et al., 2019; Organização das Nações Unidas para a Educação, a Ciência e a Cultura, 2019; Silva et al., 2018).

Entre as causas apontadas para a prática de bullying, a aparência física, incluindo a obesidade, representa $25 \%$ dos casos de bullying no mundo. As implicações psicossociais advindas da obesidade agravam-se sobremaneira na adolescência, pois esta é uma fase em que o indivíduo deseja encontrar-se como sujeito e consolidar-se como cidadão. 0 estigma social gerado em função da obesidade, a dificuldade de aceitação da autoimagem corporal, o sentimento de fracasso e de inferioridade constituem uma barreira complexa a ser ultrapassada (Bolzan Berlese, et al., 2017; Mattos et al., 2012; Melo et al., 2011; Organização das Nações Unidas para a Educação, a Ciência e a Cultura, 2019).

Fischler afirma que uma das características dos tempos atuais é a "lipofobia", ou seja, a obsessão com o peso e uma aversão quase maníaca à obesidade, proveniente da imposição social de um padrão corporal associado ao sucesso e à felicidade. No Brasil, a busca pelo "corpo ideal" colocou o país em primeiro lugar no ranking mundial de procedimentos cirúrgicos de caráter estético, reflexo da maior cobrança estética em relação a outros países. 0 excesso de peso, numa cultura que valoriza a aparência física e padrões de beleza impulsionados pela mídia, torna o indivíduo obeso alvo para discriminações em diversos contextos, sobretudo no contexto escolar, principal local descrito para a reprodução de atos de bullying (Freitas, et al., 2019; Goldenberg, 2011).

Por isso, não é incomum que indivíduos considerados obesos sofram maior exclusão social e apresentem comportamentos que se agravam com a prática do bullying. Estudos realizados com indivíduos que sofreram bullying na infância apontam como principais danos o baixo desempenho escolar, o desenvolvimento de insônia, a piora na autoestima, a dificuldade para relacionamentos, o desenvolvimento de pensamentos suicidas e distúrbios mentais na vida adulta (Alexius et al., 2018; Costa, et al., 2012; Giacomelli, et al., 2018; Malta, et al., 2019; Silva, et al., 2018).
Delbana Pereira Rodrigues, Marianne Lira de Oliveira, Paula de Moura Lopes e Cássio Eduardo Soares Miranda
Uma vez que a prática do bullying pode ser extremamente prejudicial aos indivíduos envolvidos e que a mesma tem apresentado um aumento significativo, o objetivo deste artigo é aprofundar a discussão a respeito dessa temática a partir de uma revisão integrativa sobre as implicações do bullying na saúde mental de adolescentes obesos.

\section{MÉTODO}

Trata-se de um estudo de revisão integrativa, cujo método possibilita analisar, sintetizar e ordenar as evidências disponíveis sobre o tema, permitindo sua compreensão a partir de outros estudos independentes. Para sistematização da pesquisa, obedeceu-se às seguintes etapas: delimitação do tema e formulação da questão norteadora; definição dos descritores e estratégias de busca; avaliação dos estudos; análise e interpretação dos resultados com apresentação da revisão final (Fernandes \& Galvão, 2013).

Para a primeira etapa da revisão, elaborou-se a seguinte questão norteadora: quais as implicações do bullying sobre a saúde mental de adolescentes obesos? A partir dessa questão, foi realizada a pesquisa e seleção dos descritores que seriam utilizados na equação de busca. Os descritores selecionados foram verificados junto ao DeCS (Descritores em Ciências da Saúde) e ao MeSH (Medical Subject Headings) e, por fim, cruzados aos operadores booleanos "AND" e "OR", resultando na equação: (bullying) AND (obesity) OR (overweight) AND (adolescent). Para a classificação de adolescência, considerou-se a faixa etária estabelecida pela Organização Mundial da Saúde, de 10 a 19 anos.

As bases de dados utilizadas para o rastreamento de artigos foram: Biblioteca Virtual em Saúde (BVS), United States National Library of Medicine (PubMed) e Scientifc Electronic Library Online (SciELO). Além das buscas nas bases de dados, também foi realizada a pesquisa manual em outras fontes, a partir das referências incluídas nos artigos elegíveis, a fim de localizar publicações que atendessem aos critérios de busca e seleção e que não foram localizados nas bases escolhidas. O levantamento bibliográfico ocorreu no mês de janeiro de 2019, de forma independente, por dois pesquisadores, os quais, posteriormente, examinaram a adequação e inclusão dos periódicos para a revisão final.

Após a etapa de busca, excluiu-se os artigos duplicados, seguindo com a leitura dos títulos e resumos dos artigos pré-selecionados. Os critérios empregados para inclusão dos artigos na etapa de avaliação foram: a presença dos descritores no título ou no resumo do trabalho; artigos completos em português, inglês ou espanhol; publicados no período de 2013 a 2018. Os estudos selecionados foram ordenados e analisados rigorosamente, descartando-se aqueles que não 


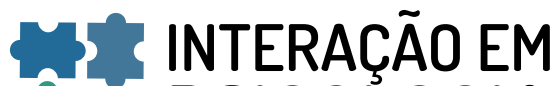 ith PSICOLOGIA}

responderam à questão norteadora e/ou que incluíram em seus resultados indivíduos pertencentes às várias faixas etárias, impossibilitando a extração apenas dos resultados pertencentes ao público-alvo da revisão. Por fim, realizou-se a análise e interpretação dos resultados e a apresentação da revisão fınal.

\section{RESULTADOS}

Foram identificadas 240 publicações a partir da estratégia de busca pré-definida. Após a exclusão de 91 artigos duplicados, foi realizada a análise dos títulos e resumos, baseada nos critérios de inclusão e exclusão definidos inicialmente. Seguida à análise, 12 artigos apresentaram-se potencialmente elegíveis e, ao final da leitura na íntegra, 05 artigos responderam à questão norteadora e foram incluídos na revisão final (Figura 01).

Do total de estudos incluídos na revisão final ( $n=05), 02$ foram realizados no Brasil, 01 no Reino Unido, 01 nos Estados Unidos e 01 na Noruega (Tabela 01).
Delbana Pereira Rodrigues, Marianne Lira de Oliveira, Paula de Moura Lopes e Cássio Eduardo Soares Miranda
Em relação aos instrumentos utilizados para a investigação de bullying em adolescentes obesos, todos os autores utilizaram a entrevista guiada por questionário semiestruturado. Bolzan et al. (2017) incluíram ainda o questionário Kidscape, a observação participante e o diário de campo. Lampard, et al. (2014) complementaram sua pesquisa com as escalas de autoestima e depressão. Sobre o local de ocorrência de bullying, os participantes relataram que a escola é o principal espaço para a perpetração do bullying, e os colegas, geralmente, são os agressores. A única forma de agressão descrita nos estudos foi a verbal, apesar de se ter conhecimento sobre a utilização de outras formas para a prática do bullying (Bolzan Berlese, et al., 2017; Lampard, et al., 2014; Øen, et al., 2018; Reece, et al., 2015; Scutti, et al., 2014).

No estudo de Bolzan Berlese, et al. (2017), os indivíduos entrevistados relataram que se sentiam excluídos e se excluíam de atividades que envolviam a interação com outros colegas, agravando o sentimento de insegurança em relação ao próprio corpo e diminuindo as possibilidades de contato social. Essa exclusão foi narrada tanto no ambiente escolar

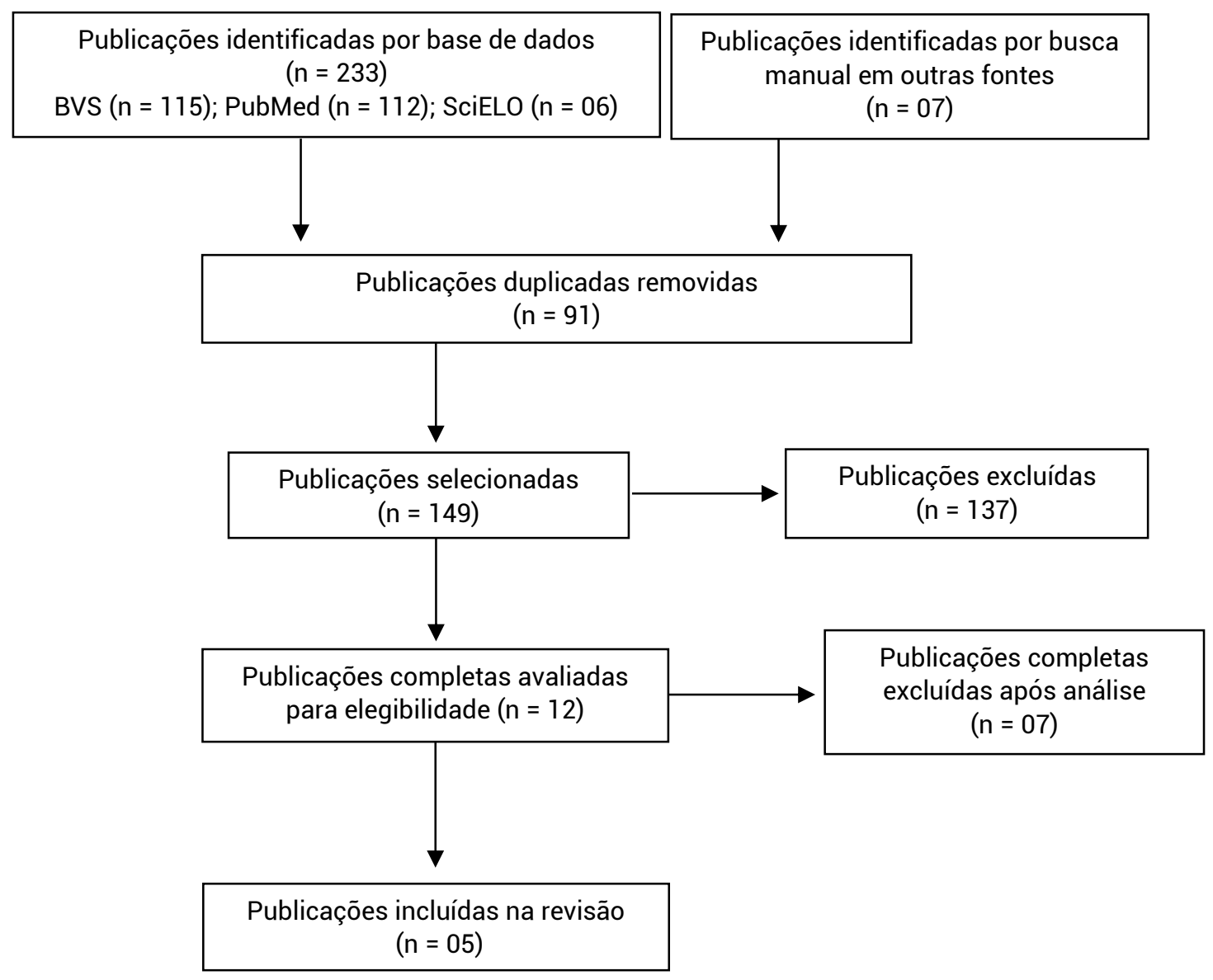

Figura 01. Protocolo de busca e seleção de artigos 
Tabela 01. Caracterização dos estudos selecionados

\begin{tabular}{|c|c|c|c|c|c|}
\hline Título & Autor/Ano & Local & Público & Objetivo & Impacto \\
\hline $\begin{array}{l}\text { Bullying e violência } \\
\text { social: Vivência de } \\
\text { adolescentes } \\
\text { obesos }\end{array}$ & $\begin{array}{c}\text { Bolzan Berlese, } \\
\text { et al. (2017) }\end{array}$ & $\begin{array}{l}\text { Rio Grande } \\
\text { do Sul - } \\
\text { Brasil }\end{array}$ & $\begin{array}{l}21 \text { adolescentes obesos } \\
\text { em tratamento no } \\
\text { ambulatório de } \\
\text { sobrepeso e obesidade } \\
\text { de um hospital de alta } \\
\text { complexidade }\end{array}$ & $\begin{array}{l}\text { Desvelar a violência de } \\
\text { bullying e violência social } \\
\text { por adolescentes obesos }\end{array}$ & $\begin{array}{l}\text { Vergonha e } \\
\text { dificuldade em } \\
\text { iniciar relações } \\
\text { sociais }\end{array}$ \\
\hline $\begin{array}{l}\text { Weight-Related } \\
\text { Teasing in the } \\
\text { School Environment: } \\
\text { Associations with } \\
\text { Psychosocial Health } \\
\text { and Weight Control } \\
\text { Practices Among } \\
\text { Adolescent Boys } \\
\text { and Girls }\end{array}$ & $\begin{array}{l}\text { Lampard, } \\
\text { et al. (2014) }\end{array}$ & $\begin{array}{l}\text { Minnesota - } \\
\text { Estados } \\
\text { Unidos }\end{array}$ & $\begin{array}{c}2.793 \text { adolescentes de } \\
20 \text { escolas públicas de } \\
\text { ensino fundamental e } \\
\text { médio }\end{array}$ & $\begin{array}{l}\text { Determinar a associação } \\
\text { entre a prevalência no nível } \\
\text { escolar de provocações } \\
\text { relacionadas ao peso e } \\
\text { fatores psicossociais, } \\
\text { insatisfação corporal e } \\
\text { comportamentos de } \\
\text { controle de peso em } \\
\text { adolescentes }\end{array}$ & $\begin{array}{l}\text { Baixa autoestima } \\
\text { nas meninas e } \\
\text { sintomas } \\
\text { depressivos nos } \\
\text { meninos }\end{array}$ \\
\hline $\begin{array}{l}\text { 'I just don't want to } \\
\text { get bullied anymore, } \\
\text { then I can lead a } \\
\text { normal life'; Insights } \\
\text { into life as an obese } \\
\text { adolescent and their } \\
\text { views on obesity } \\
\text { treatment }\end{array}$ & $\begin{array}{l}\text { Reece, et al. } \\
\text { (2015) }\end{array}$ & $\begin{array}{l}\text { South } \\
\text { Yorkshire - } \\
\text { Reino Unido }\end{array}$ & $\begin{array}{l}12 \text { participantes de } \\
\text { programas } \\
\text { comunitários para } \\
\text { controle de peso }\end{array}$ & $\begin{array}{l}\text { Explorar a experiência } \\
\text { adolescente de conviver } \\
\text { com a obesidade e seu } \\
\text { engajamento com } \\
\text { tratamentos de obesidade }\end{array}$ & $\begin{array}{l}\text { Tristeza, ansiedade } \\
\text { e sentimentos de } \\
\text { baixa autoestima e } \\
\text { exclusão social }\end{array}$ \\
\hline $\begin{array}{l}\text { O enfrentamento do } \\
\text { adolescente obeso: } \\
\text { a insatisfação com a } \\
\text { imagem corporal e o } \\
\text { bullying }\end{array}$ & $\begin{array}{l}\text { Scutti, et al. } \\
(2014)\end{array}$ & $\begin{array}{c}\text { São Paulo - } \\
\text { Brasil }\end{array}$ & $\begin{array}{c}20 \text { adolescentes em } \\
\text { tratamento no } \\
\text { Ambulatório de } \\
\text { Endocrinologia Infantil } \\
\text { do Conjunto Hospitalar } \\
\text { de Sorocaba }\end{array}$ & $\begin{array}{c}\text { Analisar a insatisfação } \\
\text { corporal de adolescentes } \\
\text { obesos de } 10 \text { a } 15 \text { anos e } \\
\text { possíveis associações ou } \\
\text { correlações da imagem } \\
\text { corporal com a } \\
\text { discriminação por bullying }\end{array}$ & Angústia e tristeza \\
\hline $\begin{array}{c}\text { Adolescents' } \\
\text { perspectives on } \\
\text { everyday life with } \\
\text { obesity: a qualitative } \\
\text { study }\end{array}$ & $\begin{array}{l}\text { Øen, } \\
\text { Kvilhaugsvik, } \\
\text { Eldal \& Anne- } \\
\text { Grethe (2018) }\end{array}$ & Noruega & $\begin{array}{l}05 \text { adolescentes que } \\
\text { buscaram os serviços } \\
\text { de saúde para } \\
\text { tratamento da } \\
\text { obesidade }\end{array}$ & $\begin{array}{c}\text { Compreender as } \\
\text { perspectivas e experiências } \\
\text { de vida de adolescentes } \\
\text { que vivem com obesidade }\end{array}$ & $\begin{array}{c}\text { Angústia, } \\
\text { insegurança para } \\
\text { se relacionar, } \\
\text { vergonha, tristeza e } \\
\text { sentimentos de } \\
\text { desesperança }\end{array}$ \\
\hline
\end{tabular}

quanto no contexto familiar. Na família, o irmão foi apontado como principal perpetrador de bullying, agravado pelo silêncio dos pais diante dessas situações. 0 autor sugere a autoexclusão na escola como uma ampliação dessa conduta em ambiente familiar.

Nas entrevistas realizadas por Reece, et al. (2015), observou-se que a reação para o bullying sofrido, em alguns casos, repercutia em modificações temporárias e radicais na relação com a comida, representada por momentos de jejuns ou no aumento da ingestão alimentar, como maneira de gerenciar ou responder ao bullying. Três participantes também referiram desejar perder peso apenas para libertarem-se de episódios de bullying e serem socialmente aceitos. Scutti, et al. (2014) constataram a forte relação entre excesso de peso e insatisfação pessoal, sendo que $95 \%$ dos entrevistados apresentaram algum grau de insatisfação com a imagem corporal. No estudo de Øen, et al. (2018), os adolescentes relataram que se sentiam mais vulneráveis pelo fato de serem "diferentes", expressando um forte desejo de serem semelhantes às outras pessoas. Eles descreveram também a ausência e 


\section{H* INTERACÃO EM L PSICOLOGIA}

necessidade de uma rede de apoio que envolva amigos e familiares, uma vez que a convivência com a obesidade foi caracterizada como algo extremamente difícil.

\section{DISCUSSÃO}

O presente estudo teve por objetivo aprofundar a discussão a respeito das implicações do bullying na saúde mental de adolescentes obesos. O bullying, enquanto forma de violência direcionada aos indivíduos obesos, constitui um tema complexo, presente no meio social e que traz consigo diversos danos: físicos, morais e psicossociais. Sua consequência primária é o dano moral, ou seja, aquele que se traduz em sofrimento imediato, decorrente da agressão sofrida (Bolzan Berlese, et al., 2017).

0 bullying pode ser externado de várias maneiras, e todas elas colaboram para que a vítima se sinta desvalorizada enquanto sujeito e sofra em relação ao seu reconhecimento social. Das categorias pesquisadas por Bolzan Berlese, et al. (2017), a agressão verbal representou 95\% das formas com que o bullying era praticado entre os adolescentes. Estudo realizado por Silva, et al. (2019) afirmou que o bullying verbal foi a forma de violência que mais vitimizou adolescentes brasileiros no período de 2009 a 2015 e foi um dos comportamentos com maior tendência de aumento. Uma possível justificativa seria o fato de pais e professores associarem tal conduta como algo inerente à faixa etária em questão, além da ausência de ações claras para o enfrentamento do bullying.

Lampard, et al. (2014) constataram em sua pesquisa que $27 \%$ dos alunos sofreram provocações relacionadas ao peso. No estudo de Scutti, et al. (2014), 50\% dos adolescentes afirmaram ter apelidos na escola e algumas das expressões mais utilizadas foram: "Baleia", "Me chamam de sanfona", "Xingam de baleia", "Me zoam porque eu sou gorda", "Traquinas" e "Tenho, mas falei, pode chamar eu de gordinho". Do total de adolescentes que relataram ter apelido, $40 \%$ afirmaram que isso incomoda, demostrando a necessidade de maior atenção por parte dos responsáveis legais e educadores para o reconhecimento desses eventos. A exposição ao bullying na adolescência tende a ser agravada por vários fatores, tais como a fragilidade psicológica característica dessa fase, o processo de formação de personalidade e a ausência da sensação de pertencimento a um grupo.

Estudos mostram que as provocações geradas em decorrência do peso são uma forma específica de bullying, que contribui para a insatisfação corporal do adolescente e para os comportamentos de controle de peso. Uma pesquisa feita por Leme \& Philippi (2013) com 159 adolescentes do sexo feminino concluiu que as provocações relativas à imagem corporal estavam associadas ao risco de desenvolver comportamen-
Delbana Pereira Rodrigues, Marianne Lira de Oliveira, Paula de Moura Lopes e Cássio Eduardo Soares Miranda tos não saudáveis para controle de peso, entre eles, a indução ao vômito e a ingestão de laxantes. Esse resultado foi similar ao observado por Duca, et al. (2010), que avaliou 5.028 adolescentes, de ambos os sexos, estudantes de escolas públicas de Santa Catarina. Os comportamentos praticados pelos pesquisados incluíam a indução ao vômito, a omissão de refeições e a ingestão de medicamentos para emagrecimento (Lampard, et al., 2014).

A literatura afırma que a exposição ao bullying e a outros tipos de violência contra adolescentes provocam sintomas persistentes que afetam o campo psicológico e se estendem ao campo físico. A revisão mostrou que a saúde mental dos adolescentes obesos, vítimas de bullying, é afetada de tal forma que estes vivenciam sentimentos de exclusão social, vergonha, insegurança emocional, baixa autoestima, sintomas depressivos, tristeza e angústia. De acordo com Malta et al. (2019), os sintomas descritos podem agravar-se na vida adulta, tornando o indivíduo mais propenso ao desenvolvimento de distúrbios mentais e prática de atitudes-limite, como o suicídio. Logo, encarar as consequências do bullying é de interesse social (Souza, et al., 2018).

Em relação ao local para perpetração do bullying, o ambiente escolar foi reconhecido como um dos principais locais e onde as consequências deste foram identificadas, uma vez que o ambiente escolar é um espaço de interação entre pares com fragilidades corporais semelhantes, faixas etárias similares e onde é reconhecida a tendência à reverberação e reprodução das agressões às quais os escolares foram submetidos. 0 bullying também foi identificado no ambiente familiar, concomitante a outros tipos de violência que são negligenciadas, devido à ideia de família enquanto instituição de proteção e defesa contra maus-tratos. Somado a isto, as agressões perpetradas por familiares por vezes são legitimadas no convívio social, defendidas como modo de educar e corrigir condutas indesejadas (Barros \& Freitas, 2015; Silva, 2012).

Dessa forma, o bullying se apresenta, enquanto expressão social da obesidade, carregado de significados e representações sociais do corpo que vão além do peso corporal físico e individual. Fica evidente que a cobrança social pela cultura "ao corpo perfeito" tende a afetar em maior proporção os adolescentes que estão em processo de formação corporal, resultando em processos de emagrecimento doentio ou, até mesmo, aumento na ingestão de alimentos como meio de compensação do sofrimento (Bolzan Berlese, et al., 2017).

\section{CONSIDERAÇÕES FINAIS}

O bullying, de forma geral, traz grandes prejuízos às suas vítimas. Na revisão apresentada, foi possível constatar a existência de sentimentos negativos vivenciados por ado- 


\section{Asurearion PSICOLOGIA}

lescentes obesos e que são vítimas de bullying. Tais achados evidenciam uma certa urgência para o desenvolvimento de ações permanentes de enfrentamento ao bullying, a fim de minimizar o risco de danos à saúde mental e a alimentação de comportamentos negativos.

Nesse contexto, a escola em particular, por ser o principal local mencionado para ocorrência desses eventos, precisa estar atenta e elaborar estratégias que incluam a participação de escolares, familiares, professores e profissionais de diversas áreas para a promoção do convívio saudável. Sendo assim, a revisão apresentada não é o fim em si mesma, mas um desafio para impulsionar as iniciativas para redução do bullying escolar.

\section{CONTRIBUIÇÃO DE CADA AUTOR}

Certificamos que todos os autores participaram suficientemente do trabalho para tornar pública sua responsabilidade pelo conteúdo. A contribuição de cada autor pode ser atribuída como se segue:

D. P. R. e M. L. O. contribuíram para a idealização do estudo, aplicação da metodologia, análise dos dados e redação do manuscrito. P. M. L. contribuiu para a redação do manuscrito. C. E. S. M. foi responsável pela supervisão e revisão do manuscrito.

\section{DECLARAÇÃO DE CONFLITOS DE INTERESSES}

Os autores declaram que não há conflitos de interesses no manuscrito submetido.

\section{REFERÊNCIAS}

Alexius, S. L., Mocellin, M. C., Corrêa, E. N., Neves, J. d., Vasconcelos, F. d., \& Corso, A. C. (2018). Evidências da associação entre atributos individuais e bullying: um estudo transversal em adolescentes de Florianópolis, Santa Catarina, Brasil. Cadernos de Saúde Pública, 34(12), 1-14. https://doi.org/10.1590/0102-311x00118617

Barros, A. S., \& Freitas, M. d. (2015). Violência doméstica contra crianças e adolescentes: consequências e estratégias de prevenção com pais agressores. Pensando famílias, 19(2), 102-114. http://pepsic.bvsalud.org/scielo.php?script=sci_arttext\&pid=S1679-494X2015000200009

Bolzan Berlese, D., Roese Sanfelice, G., Bolzan Berlese, D., \& Sidegum Renner, J. (2017). Bullying e violência social: Vivência de adolescentes obesos. Revista Latinoamericana de Ciencias Sociales, Niñez y Juventud, 15(1), 491-503. https://doi.org/10.11600/1692715x.1513111042016
Delbana Pereira Rodrigues, Marianne Lira de Oliveira, Paula de Moura Lopes e Cássio Eduardo Soares Miranda
Costa, M. A., Souza, M. A., \& Oliveira, V. M. (2012). Obesidade infantil e bullying: a ótica dos professores. Educação e Pesquisa, 38(3), 653-665. https://doi.org 10.1590/S151797022012005000017

Duca, G. F., Garcia, L. M., Sousa, T. F., Oliveira, E. S., \& Nahas, M. V. (2010). Insatisfação com o peso corporal e fatores associados em adolescentes. Revista paulista de pediatria, 28(4), 340-346. https://doi.org/10.1590/S010305822010000400009

Fernandes, A. F., \& Galvão, C. M. (2013). Métodos de revisão: Não podemos banalizar! Revista da rede de enfermagem do nordeste, 14(1), 1-2. http://periodicos.ufc.br/rene/article/ view/3313/2551

Freitas, R. G., Pereira, L. L., Alves, P. S., \& Oliveira, M. R. (2019). Lipofobia, disciplinamento do corpo e produção de valor. Conexões, 1-17. https://doi.org/10.20396/conex. v17i0.8655108

Giacomelli, S., Moreira, G. S., Santos, G. C., Guimarães, B. F., \& Rodrigues, A. M. (2018). Obesidade e Bullying: Um enfrentamento da imagem corporal. [Anais de evento]. Congresso Interdisciplinar - Ciência para a Redução das Desigualdades. Faculdade Evangélica de Goianésia, Anápolis. http://anais. unievangelica.edu.br/index.php/cifaeg/article/view/2003

Goldenberg, M. (2011). Gênero, "o Corpo" e a "Imitação Prestigiosa" na cultura Brasileira. Saúde e Sociedade, 20(3), 543553. https://doi.org/10.1590/S0104-12902011000300002

Lampard, A. M., MacLehose, R. F., Eisenberg, M. E., Neumark-Sztainer, D., \& Davison, K. K. (2014). Weight-related teasing in the school environment: Associations with psychosocial health and weight control practices among adolescent boys and girls. Journal of Youth and Adolescence, 43(10), 1770-1780. https://doi.org/10.1007/s10964-013-0086-3

Leme, A. C., \& Philippi, S. T. (2013). Provocações e comportamentos para controle de peso em adolescentes do sexo feminino. Revista Paulista de Pediatria, 31(04), 431-436. https://doi.org/10.1590/S0103-05822013000400003

Malta, D. C., Mello, F. C., Prado, R. R., Sá, A. C., Marinho, F., Pinto, I. V., Silva, M. M. A., \& Silva, M. A. (2019). Prevalência de bullying e fatores associados em escolares brasileiros, 2015. Ciência \& Saúde Coletiva, 24(4), 1359-1368. https:// doi.org/10.1590/1413-81232018244.15492017

Mattos, R. d., Perfeito, R., Carvalho, M. C., \& Retondar, J. (2012). Obesidade e bullying na infância e adolescência: o estigma da gordura. Demetra: Alimentação, Nutrição \& Saúde, 7(2), 71-84. https://doi.org/10.12957/demetra.2012.3330

Melo, T. R., Jansen, A. K., Pinto, R. d., Morales, R. R., Morales, N. M., Prado, M. M., \& Silva, C. H. (2011). Qualidade de vida de cuidadores de crianças e adolescentes com sobrepeso ou obesidade. Revista da Escola de Enfermagem da USP, 45(2), 319-326. https://doi.org/10.1590/S008062342011000200003 


\section{- IN INERACÃO EM LFICOLOGIA}

Øen, G., Kvilhaugsvik, B., Eldal, K., \& Anne-Grethe, H. (2018). Adolescents' perspectives on everyday life with obesity: A qualitative study. International Journal of Qualitative Studies on Health and Well-being, 13(1), 1-12. https://doi.org /10.1080/17482631.2018.1479581

Reece, L. J., Bissell, P., \& Copeland, R. J. (2015). 'I just don't want to get bullied anymore, then I can lead a normal life'; Insights into life as an obese adolescent and their views on obesity treatment. Health Expectations, 19(4), 897-907. https://doi.org/10.1111/hex.12385

Scutti, C. S., Seo, G. Y., Amadeu, R. S., \& Sampaio, R. F. (2014). $O$ enfrentamento do adolescente obeso: A insatisfação com a imagem corporal e o bullying. Revista da Faculdade de Ciências Médicas de Sorocaba, 16(3), 130-133. https:// revistas.pucsp.br/RFCMS/article/view/15188

Silva, A. N., Marques, E. S., Peres, M. F., \& Azeredo, C. M. (2019). Tendência de bullying verbal, violência doméstica e envolvimento em brigas com armas entre adolescentes das capitais brasileiras de 2009 a 2015. Cadernos de Saúde Pública, 35(11), 1-16. https://doi.org/10.1590/ 0102-311x00195118
Delbana Pereira Rodrigues, Marianne Lira de Oliveira, Paula de Moura Lopes e Cássio Eduardo Soares Miranda

Silva, B. R., Silva, B. R., Passos, M. H., Soares, F. C., Valença, P. A., Menezes, V. A., Colares, V., \& Santos, C. d. (2018). Autopercepção negativa de saúde associada à violência escolar em adolescentes. Ciência \& Saúde Coletiva, 23(9), 2909-2916. https://doi.org/10.1590/141381232018239.12962018

Silva, C. G. (2012). A violência doméstica e sua influência na aprendizagem: um estudo exploratório no centro de integração familiar (ceifar). Revista Tarrafa, 01, 96-106. http:// www.uneb.br/tarrafa/files/2012/10/a-viol\%c3\%aancia-dom\%c3\%a9stica-e-sua-influ\%c3\%aancia-na-aprendizagem.pdf

Souza, C. M., Vizzotto, M. M., \& Gomes, M. B. (2018). Relação entre violência familiar e transtorno de estresse pós-traumático. Psicologia, Saúde \& Doenças, 19(2), 222-233. https://doi.org/10.15309/18psd190205

Organização das Nações Unidas para a Educação, a Ciência e a Cultura. (2019). Violência escolar e bullying: relatório sobre a situação mundial. Brasília: Organização das Nações Unidas para a Educação, a Ciência e a Cultura. https:// unesdoc.unesco.org/ark:/48223/pf0000368092

Data de submissão: $24 / 01 / 2020$ Primeira decisão editorial: $25 / 04 / 2020$ Aceite em 19/06/2020 